\title{
Controlling Active Brownian Particles in Complex Settings
}

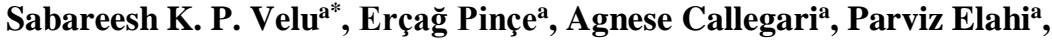 \\ Sylvain Gigan ${ }^{\mathrm{b}}$, Giovanni Volpe ${ }^{\mathrm{a}, \mathrm{c}, \mathrm{d}}$, Giorgio Volpe ${ }^{\mathrm{e}}$ \\ ${ }^{a}$ Department of Physics, Bilkent University, Çankaya, 06800 Ankara, Turkey. \\ ${ }^{b}$ Laboratoire Kastler Brossel, Université Pierre et Marie Curie, École Normale Q1 Supérieure, CNRS, College de France, 24 rue Lhomond, \\ 75005 Paris, France. \\ ${ }^{c}$ UNAM-National Nanotechnology Research Center, Bilkent University, Çankaya, 06800 Ankara, Turkey. \\ ${ }^{d}$ Physics Department, University of Gothenburg, 40530 Gothenburg, Sweden \\ ${ }^{e}$ Department of Chemistry, University College London, 20 Gordon Street, London WC1H OAJ, UK. \\ *Corresponding author: sabareesh@fen.bilkent.edu.tr
}

\begin{abstract}
We show active Brownian particles (passive Brownian particles in a bacterial bath) switches between two long-term behaviors, i.e. gathering and dispersal of individuals, in response to the statistical properties of the underlying optical potential.

OCIS codes: (350.4855) Optical tweezers or optical manipulation
\end{abstract}

Brownian particles undergo stochastic and random motion due to the thermal agitation in the surrounding medium. In recent years, a lot of attention has been devoted to the study of active Brownian particles from the biology and physics communities. These active Brownian particles, often referred to as microswimmers, are able to propel themselves, exhibiting an interplay between random fluctuations and active swimming that drives them into a far-from-equilibrium state. Thanks to this property, they feature a series of novel behaviors that are not attainable by passive particles, including, for example, self-assembly, swarming, and the emergence of other collective properties. In this work [1], I will present recent experimental results where we show that the presence of disorder potential has an influence on the active particles that switches between gathering and dispersal. Interestingly, the statistical properties of the disorder potential allow us to dynamically control the long-term collective behaviour of the active matter system (Figure 1). Beyond these fundamental interests, these results are significant to engineer autonomous agents interacting with realistic (complex and crowded) surroundings, for example, artificial microswimmers capable of localizing, picking up and delivering nanoscopic cargos in catalysis, bioremediation, chemical sensing and drug delivery.
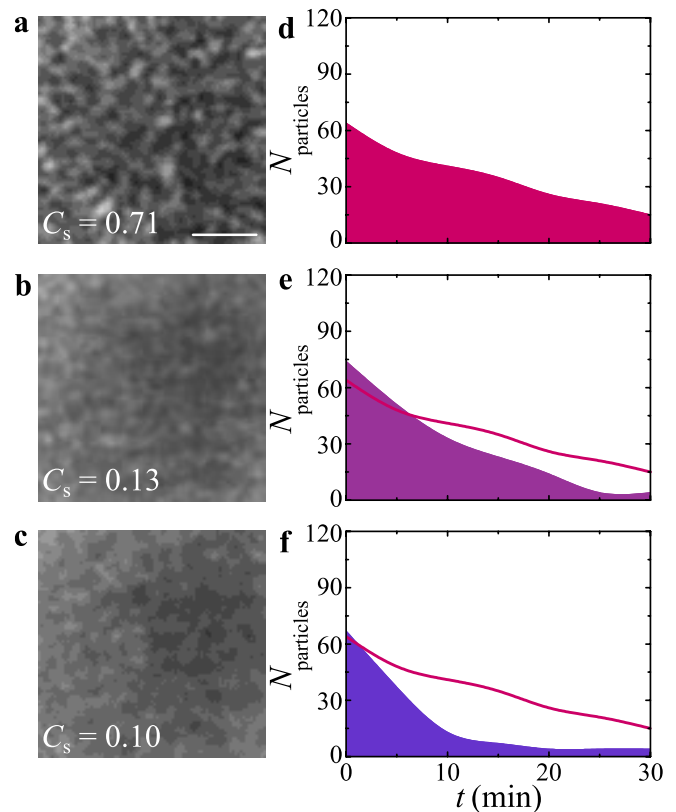
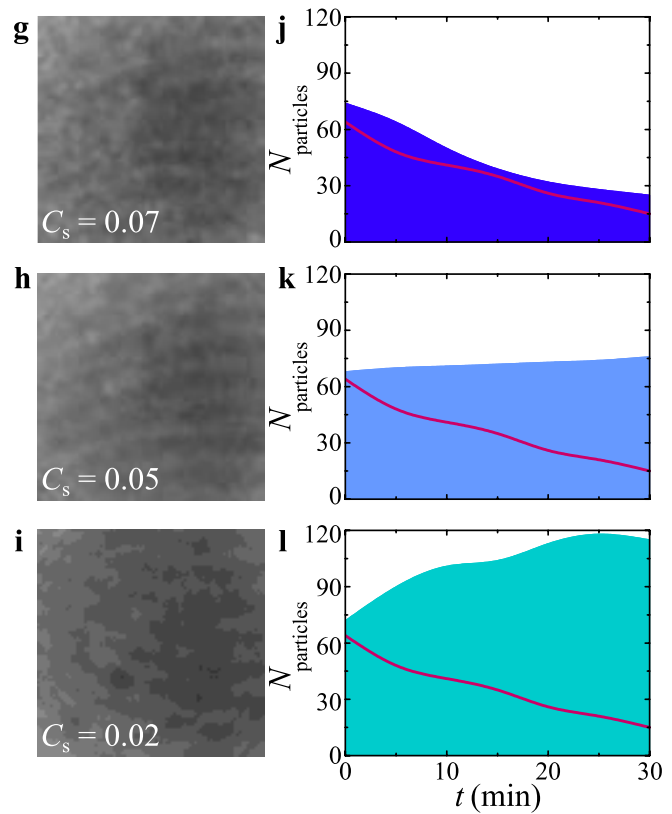

Fig. 1. Controlling the long-term behavior (dispersal-to-gathering) of the active Brownian particles by dynamically controlling the roughness of the potential (disorder-to-smooth). Adapted from Ref. 1.

\section{References}

[1] Erçağ Pinçe, Sabareesh K.P. Velu, Agnese Callegari, Parviz Elahi, Sylvain Gigan, Giovanni Volpe, Giorgio Volpe, "Disorder-mediated crowd control in an active matter system," Nature Communications 7, 10907 (2016). 